\title{
Studying the Pharmaceutical Film Coating Process with Terahertz Sensing, Optical Coherence Tomography and Numerical Modelling
}

\author{
Hungyen Lin ${ }^{1,2}$, Yue Dong ${ }^{3}$, Chunlei Pei $^{4}$, Bryan M. Williams ${ }^{5}$, Daniel Markl ${ }^{2}$, Yalin Zheng ${ }^{5}$, James \\ A. Elliott ${ }^{4}$, Yaochun Shen ${ }^{3}$, and J. Axel Zeitler ${ }^{2}$ \\ ${ }^{1}$ Department of Engineering, Lancaster University, UK \\ ${ }^{2}$ Department of Chemical Engineering and Biotechnology, University of Cambridge, UK \\ ${ }^{3}$ Department of Electrical Engineering and Electronics, University of Liverpool, UK \\ ${ }^{4}$ Department of Material Science and Metallurgy, University of Cambridge, UK \\ ${ }^{5}$ Department of Eye and Vision Science, University of Liverpool, UK
}

\begin{abstract}
Terahertz in-line sensing was successfully demonstrated on a production scale setting for measuring the coating thickness of individual pharmaceutical tablets during the film coating process. This paper reports on our recent research progress to combine terahertz in-line sensing, optical coherence tomography and numerical modelling in a lab scale setting to better understand the pharmaceutical film coating process.
\end{abstract}

\section{INTRODUCTION}

$\mathrm{P}$ HARMACEUTICAL film coatings are typically polymeric films formed from an aqueous latex dispersion that are used for the purpose of prolonging shelf life of the active ingredient and, improving the aesthetics of the dosage forms and in advanced dosage forms, the coatings also serve a functional purpose such as in active coating and controlled release.

Film coating is typically performed in large batches and owing to the process complexities stemming from the large number of process parameters broadly classified as: dosage form properties (shape, size), process conditions (rotation speed, temperature, humidity, airflow) and device parameters (baffle design, nozzle location), the resulting product shows a very heterogeneous coating distribution thus limiting the applicability of advanced dosage forms. To enhance process understanding, various non-destructive in-line techniques have been demonstrated. Whilst near-IR [1] and Raman spectroscopy [2] have proven to be popular techniques, largely because of the wide availability of sources and detectors, these techniques measure coating thickness indirectly and thus require time-consuming calibration models to be constructed. Furthermore, owing to the inherent high sensitivity, temporal and spatial averaging of the measurements are necessary and therefore information specific to each of the dosage form, such as the inter-tablet coating variation, cannot be captured.

In-line terahertz sensing [3] and optical coherence tomography (OCT) [4] are relatively new sensing modalities that show tremendous potential for pharmaceutical coating investigations. In particular, terahertz sensing is well suited for tablet dosage forms with coating thickness in the range of 40 $\mu \mathrm{m}-1 \mathrm{~mm}$, whereas OCT is applicable to tablets and pellets for coating thicknesses of $10-80 \mu \mathrm{m}$. By combining these two promising techniques together in an in-line setting, in-situ and real-time film coating thickness information can be obtained that can subsequently be consolidated with numerical models in order to develop a mechanistic understanding of the film coating process. The aim of this paper is therefore to report on the recent research progress.

\section{METHODS}

A 1 litre laboratory-sized perforated coating pan was designed and manufactured as shown in Figure 1. We have now modified the coating pan to a fully contained lab scale coating unit where we can perform batch spray coating of tablets. The coater includes relevant sensors to monitor and record process parameters such as airflow, temperature, humidity and pressure. In a manner similar that described in [3], the coating unit has been successfully coupled to the TPI Imaga 2000 (TeraView Ltd., Cambridge, UK), where TPI operates at an acquisition rate of $30 \mathrm{~Hz}$ to measure the coating thicknesses of tablets. In parallel, a fiber based spectral domain OCT system operating at $840 \mathrm{~nm}$ with kilohertz acquisition rate has been integrated into the coating unit to perform in-line sensing. In the film coating process, a sustained coating formulation that consists Kollicoat SR30D (BASF, Ludwigshafen, Germany) is applied to biconvex cores. Based on the previously published analysis algorithm $[3,5]$, the $\mathrm{THz}$ measurements acquired during the film coating process are automatically processed with Matlab. Similarly, the developed data processing algorithm using image segmentation is applied to automatically process the OCT measurements [6]. In contrast to earlier works [3,7], offline measurements were only taken before and after the coating process so as to not risk the coating unit out of physical alignment with the TPI for THz in-line sensing.

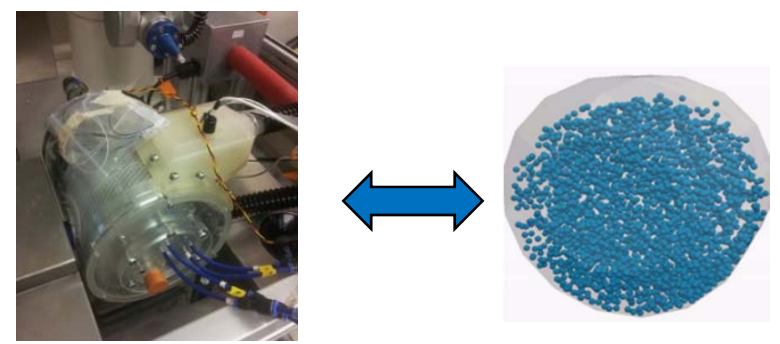

Fig. 1. A numerical model for the pharmaceutical coating process consolidated with process feedback information in the form of film coating thickness measured in-situ by in-line SD-OCT and terahertz sensing.

\section{RESULTS}

Using the refractive index of the coating material at the $\mathrm{THz}$ and optical frequencies obtained by measuring the film coating thickness of a randomly selected coated tablet with X-ray computed microtomography, the absolute film coating thickness that is independently measured with OCT and $\mathrm{THz}$ throughout the process can be compared against one another as shown in Fig. 2. As can be expected, there is a steady increase 
in the film coating thickness observed with both sensing modalities. Furthermore, thin coatings can be resolved with the shorter wavelength OCT where measurement starts as early as in the first 10 minutes of the coating process. Meanwhile, $\mathrm{THz}$ measurements, owing to the picosecond pulse duration, can resolve thicker coatings until the process endpoint. The thickness resolution of $50 \mu \mathrm{m}$ achieved with $\mathrm{THz}$ in this work is greater than the previously published values of $30-40 \mu \mathrm{m}$ due to the additional selection criteria used in the data processing algorithm. Nevertheless, the film coating thickness measured by $\mathrm{THz}$ at close to the process endpoint lie within the error bar of the offline thickness measurement with TPI. Also can be observed is the period of overlap in the acquired film coating thickness by the respective methods during the time interval between 50 to 150 minutes. The increased thickness variability measured by OCT particularly after 100 minutes can be validated against the acquired cross-sectional images or Bscans of the imaged coatings in order to assess the physicality of the measurement. This is made possible because of the two orders of magnitude higher data acquisition rate used. An example B-scan showing a tablet measurement is shown in Fig. 3.

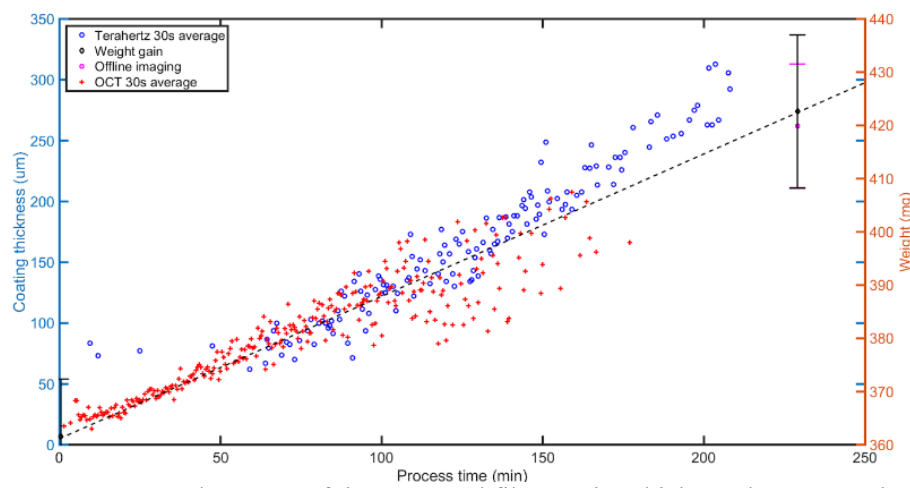

Fig. 2. 30 second average of the measured film coating thickness by OCT and $\mathrm{THz}$ in-line sensing as a function of the coating time.

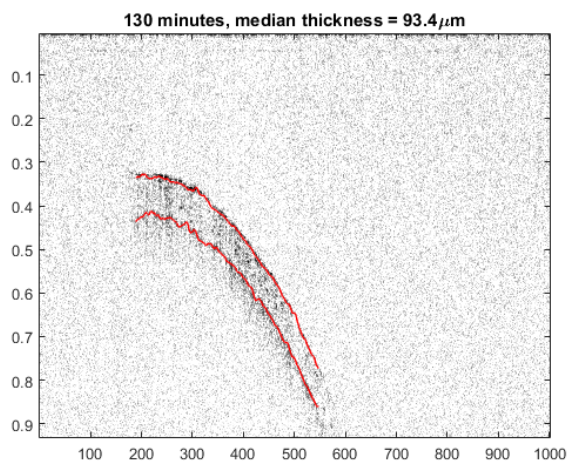

Fig. 3. An example B-scan acquired by OCT during the film coating process showing the identified film coating interfaces for thickness quantification.

\section{SUMMARY}

We have successfully modified the lab-scale coating unit such that we can perform combined OCT and $\mathrm{THz}$ in-line sensing in order to study the pharmaceutical film coating process. Future work aims to consolidate the film coating measurements acquired with numerical models to explain the observed changes in the coating uniformity throughout the process.

\section{ACKNOWLEDGEMENT}

The authors would like to acknowledge the financial support from UK EPSRC Research Grant EP/L019787/1 and EP/L019922/1. The authors acknowledge BASF for providing the materials used in this study, Colorcon Ltd. (Dartford, UK) for coating process recommendations and Huettlin GmbH (Bosch Packaging Technology, Schopfheim, Germany) for advices on the coating unit design. HL also acknowledges travel support from Joy Welch Educational Charitable Trust.

\section{REFERENCES}

[1]. J.D. Perez-Ramos, W.P. Findlay, G. Peck, K.R. Morris, "Quantitative analysis of film coating in a pan coater based on in-line sensor measurements", AAPS PharmSciTech, vol. 6, 2005.

[2]. M. Wirges, A. Funke, P. Serno, K. Knop, P. Kleinebudde, "Development and in-line validation of a Process Analytical Technology to facilitate the scale up of coating processes", J. Pharm. Biomed. Anal., vol. 78-79, pp. 57-64, 2013. [3]. R. K. May, M. J. Evans, S. Zhong, I. Warr, L. F. Gladden, Y. Shen, and J. A. Zeitler, "Terahertz In-Line Sensor for Direct Coating Thickness Measurement of Individual Tablets During Film Coating in Real-Time", $J$. Pharm Sci. vol. 100, pp. 1535, 2011.

[4]. S. Zhong, Y-C Shen, L Ho, RK May, JA Zeitler, M. Evans, Taday PF, Pepper M, Rades T, Gordon KC, Müller R, Kleinebudde P., "Nondestructive Testing of Pharmaceutical Coatings by Terahertz Pulsed Imaging and Optical Coherency tomography", Optics and Lasers in Engineering, vol. 49(3), pp. 361-365, 2011.

[5]. H. Lin, R.K. May, M. Evans, S. Zhong, L.F. Gladden, Y.C. Shen and J.A. Zeitler, "Impact of Processing Conditions on Inter-tablet Coating Thickness Variations Measured by Terahertz In-Line Sensing", J. Pharm. Sci., vol. 104(8), pp. 2513-2522, 2015.

[6]. D. Williams, Y. Zheng, P.G. Davey, F. Bao, M. Shen, and A. Elsheikh, "Reconstruction of 3D surface maps from anterior segment optical coherence tomography images using graph theory and genetic algorithms,". Biomed. Signal Process. Control, vol. 25, pp. 91-98, 2016.

[7]. D. Markl, G. Hannesschläger, S. Sacher, M. Leitner, A. Buchsbaum, R. Pescod, T. Baele and J.G. Khinast, "In-Line Monitoring of a Pharmaceutical Pan Coating Process by Optical Coherence Tomography," J. Pharm. Sci., vol. 104(8), pp. 2531-2540, 2015. 